\title{
METODOLOGIAS PARA QUALIFICAÇÃO TECNOLÓGICA APLICADA AO DESENVOLVIMENTO DE PRODUTO: UMA REVISÃO SISTEMÁTICA
}

Pedro Martins de Oliveira ${ }^{1}$, Valter Estevão Beal²

${ }^{1}$ Centro universitário SENAI CIMATEC, Avenida Orlando gomes 1845, Salvador-BA; pedromartins175@gmail.com

${ }^{2}$ Centro universitário SENAI CIMATEC, Avenida Orlando gomes 1845, Salvador-BA

Resumo: Este trabalho tem como objetivo caracterizar a produção científica sobre o processo de qualificação tecnológica aplicada ao desenvolvimento de produto e, para isso, foi realizada uma revisão sistemática com o objetivo de identificar os principais trabalhos na área, identificando metodologias e ferramentas aplicadas. Foram identificados 12 estudos que evidenciam o uso da qualificação tecnológica na etapa de desenvolvimento de produto, cujos resultados mostram a diversidade de metodologias para a qualificação tecnológica, com apenas um estudo utilizando de métricas relacionadas a necessidades dos clientes, demonstrando a necessidade de mais pesquisa nessa linha.

Palavras-Chave: Qualificação de tecnologias, Desenvolvimento de produto, Incertezas da Inovação.

\section{METHODOLOGIES FOR TECHNOLOGICAL QUALIFICATION APPLIED TO PRODUCT DEVELOPMENT: A SYSTEMATIC REVIEW}

\begin{abstract}
This paper aims to characterize the scientific production about the technological qualification process applied to product development. For this, a systematic review was carried out to identify the main works in the area, identifying methodologies and applied tools. Twelve studies were identified that show the use of technological qualification in the product development stage. The results shows the diversity of methodologies for technological qualification, with only one study using metrics related to customer needs, demonstrating the need for further research in this area.
\end{abstract}

Keywords: Technology Qualification, Product Development, Innovation uncertities. 


\section{INTRODUÇÃO}

O processo de desenvolvimento de produto é uma atividade criativa que requer sólidos conhecimentos em engenharia, por isso o processo de desenvolvimento integrado de produto consiste na utilização de métodos estruturados, não para limitar a criatividade da equipe de projeto, mas para entender as necessidades dos clientes e trazer um conceito que traga resultados inovadores [1]

Algumas ferramentas podem ser utilizadas para auxiliar neste processo, criando de maneira sistemática soluções para problemas cotidianos em escritórios de projeto. Esses métodos sistemáticos são chamados de DFX (design for excelency), que consiste em uma família de métodos de projeto para solucionar algum problema relacionado a um fator que ele dever ter, sendo o mais famoso deles o DFMA (Design for manufacturing and assembly), que traz regras e métodos para o desenvolvimento de um produto levando em consideração os fatores de montagem e fabricação [2].

Além dessas ferramentas é também comum o uso nos produtos de tecnologias que não foram testadas em ambiente relevante, sendo necessário um processo de qualificação da tecnologia, para isso as guias para práticas de qualificação de novas tecnologias são expostas pela DNVRP-A203. Este procedimento explica como identificar, descrever e gerenciar riscos oriundos das incertezas tecnológicas [3].

O motivo de atenção para a qualificação tecnológica é que, muitas vezes, o desenvolvimento destas tecnologias são custosos, trazem riscos para o projeto e para o produto final [4].

O estado da prática expõe diretrizes para o avanço da tecnologia em um projeto, e alguns estudos mostram ferramentas para remediar essas incertezas que são desenvolvidas no processo de desenvolvimento de produto [5]. Entretanto fica evidente a falta de literatura que relaciona os requisitos e necessidades dos clientes como métricas para o avanço da maturidade tecnológica.

O objetivo deste trabalho é analisar as contribuições das pesquisas produzidas acerca de metodologias aplicadas na qualificação tecnológica no processo de desenvolvimento de produto, com vistas em comprimento de requisitos dos clientes no processo de avanço da maturidade da tecnologia.

\section{METODOLOGIA}

Este estudo foi desenvolvido por meio de uma pesquisa bibliográfica e contemplou aspectos relacionados ao uso de métodos de qualificação tecnológica aplicada ao desenvolvimento de produto.

Trata-se de um estudo de revisão sistemática da literatura, cuja construção passou pelas seguintes etapas: identificação do objeto de estudo; formulação da questão norteadora do estudo; identificação das bases de dados a serem consultadas; definição dos descritores; definição dos critérios de inclusão e exclusão dos trabalhos; 
análise e avaliação de todos os estudos incluídos na revisão; produção de uma síntese das informações e resultados encontrados e apresentação da revisão.

As bases de dados selecionadas foram as seguintes: Science direct, IEEE explore e One petro. Os descritores escolhidos foram: "Technology qualification", "product development" e "requirements management", reordenando-os utilizando dos operadores lógicos "and" e "or".

Os critérios de inclusão foram: artigos que abordavam o processo de qualificação da tecnologia como um processo integrado ao desenvolvimento de produto, utilizando métricas comuns de confiabilidade ou de requisitos de projetos no período entre 2009 e 2019, disponibilizados na íntegra na língua inglesa ou portuguesa e na base de dados da One Petro somente artigos submetidos a revisão por pares participaram do estudo.

O processo de exclusão partiu da metodologia exposta na figura 1: exclusão dos duplicados, análise dos títulos e palavras chaves (Necessário, pois o banco de dados da One Petro não permite pesquisar somente por título e palavras chaves) e por final análise dos resumos e métricas utilizados.

Figura 1 - Sistemática de exclusão dos artigos.

\begin{tabular}{|c|c|c|c|c|c|c|}
\hline \multirow{3}{*}{$\begin{array}{l}\text { Pesquisa } \\
\text { sistemática }\end{array}$} & \multirow[t]{3}{*}{ Artigos } & \multirow{2}{*}{$\begin{array}{l}\text { Remoção dos } \\
\text { duplicados }\end{array}$} & Artigos & \multirow{2}{*}{$\begin{array}{c}\text { Análise das } \\
\text { palavras chaves e } \\
\text { título }\end{array}$} & \multirow[t]{2}{*}{ Artigos } & \multirow{2}{*}{$\begin{array}{c}\text { Análise dos } \\
\text { resumos e } \\
\text { métricas utilizadas }\end{array}$} \\
\hline & & & & & & \\
\hline & & $\begin{array}{l}\text { Artigos } \\
\text { excluídos }\end{array}$ & & \begin{tabular}{|l|l} 
Artigos \\
excluídos
\end{tabular} & & \begin{tabular}{|l|l}
$\begin{array}{l}\text { Artigos } \\
\text { excluídos }\end{array}$ \\
\end{tabular} \\
\hline
\end{tabular}

Além desses critérios de inclusão, foram inseridos critérios de qualidade para os artigos, que foram: $O$ caso pode ser aplicado em um processo de desenvolvimento de produto integrado e requisitos de projetos ou necessidades dos clientes foram métricas nos resultados.

\section{RESULTADOS E DISCUSSÃO}

A partir deste métodos ficaram 12 artigos selecionados mostrado na tabela 1. 
Tabela 1: Relação dos artigos coletados na pesquisa.

\begin{tabular}{|l|l|l|l|}
\hline Descritores & $\begin{array}{l}\text { Science direct } \\
\text { Total / Incluídos na } \\
\text { pesquisa }\end{array}$ & $\begin{array}{l}\text { IEEE } \\
\text { Total / Incluídos na } \\
\text { pesquisa }\end{array}$ & $\begin{array}{l}\text { One Petro } \\
\text { Total / Incluídos na } \\
\text { pesquisa }\end{array}$ \\
\hline $\begin{array}{l}\text { "Product development" and } \\
\text { "Technology qualification }\end{array}$ & $7 / 2$ & 0 & $1 / 0$ \\
\hline $\begin{array}{l}\text { "Technology qualification" and } \\
\text { "requirements" }\end{array}$ & $33 / 6$ & $6 / 1$ & $13 / 3$ \\
\hline Total de artigos inclusos & 8 & 1 & 3 \\
\hline
\end{tabular}

É possível observar um aumento das publicações entre 2014 e 2016 na Figura 2. Este número vem crescendo pela necessidade da indústria de óleo e gás de novas tecnologias que possam ser usadas em ambientes em maior altura de lâmina d'agua e necessitando de um método sistemático para a qualificação dessas tecnologias.

Figura 2: Número de artigos no período investigado

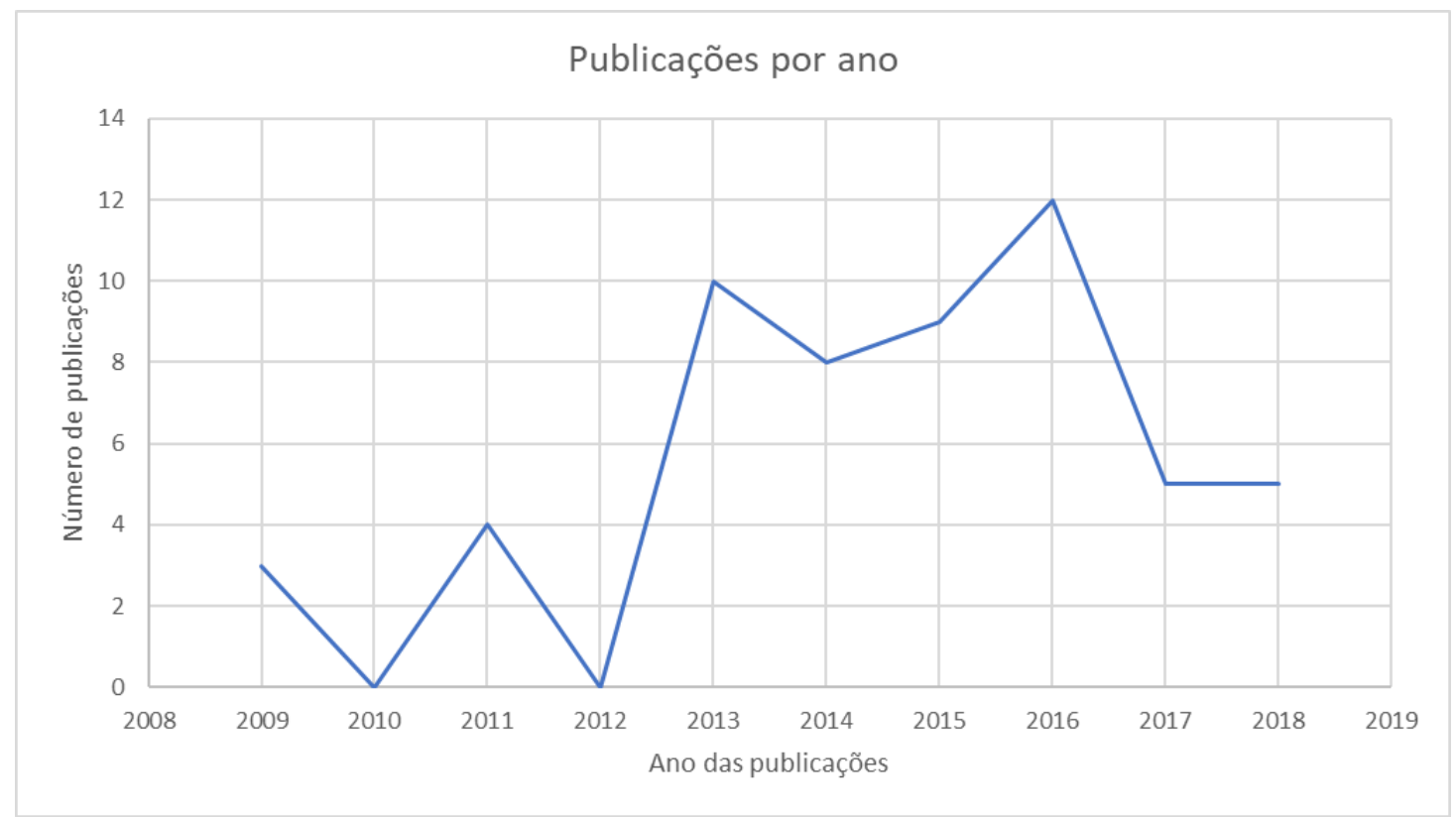

Após a análise dos artigos selecionados foi percebido a falta de consenso em processos de qualificação tecnológica aplicada a desenvolvimento de produto. Alguns trabalhos partem de uma abordagem voltada à confiabilidade utilizando de ferramentas de uso conhecido como FMEA (failure mode anlysis), enquanto outra parcela utiliza de questionários e entrevistas com especialistas para identificação da maturidade tecnológica. Poucos estudos levantam as necessidades dos clientes como métrica para o avanço da maturidade da tecnologia.

A seguir a síntese da análise dos artigos selecionados para o estudo.

E. Hessen et al, (2013) [6] utilizou das recomendações da norma Det Norske Veritas (DNV) para a qualificação de um absorvedor de dióxido de carbono em larga escala. Foi um processo baseado em risco utilizando de modelagem do processo de 
absorção como modo de prever a criticidade dos modelos preliminares desenvolvidos. $O$ artigo traz ênfase na validação destes sub-modelos que confrontam os valores disponíveis em dados experimentais da literatura e dados obtidos através dos submodelos.

R. Proskovics et al, (2016) [7] propôs uma metodologia para avaliar os riscos de subestruturas de turbinas eólicas flutuantes, $O$ autor separa em etapas, como avaliação dos riscos tecnológicos, análise da composição tecnológica, categorização da tecnologia, riscos à saúde, segurança e meio ambiente, riscos na manufatura e comercialização. O presente estudo não possuí aplicações da metodologia e nem métricas.

M. Sabet (2013) [8] propôs uma metodologia baseada em objetivo para a qualificação tecnológica utilizando de opiniões de especialistas e satisfação do comprimento desses objetivos. $\mathrm{O}$ artigo participou em dois estudos de casos na área industrial de óleo e gás; foi efetuado a coleta de dados construindo o modelo de objetivo através de coleta das informações técnicas do portfólio da indústria refinando as informações com entrevistas com especialistas.

T. Myhrvold et al. (2009) [9] propõe uma metodologia para a qualificação tecnológica de tecnologias de captura de dióxido de carbono em três fases: desenvolvimento das diretrizes, o teste das diretrizes em estudos de casos e mudança das diretrizes para uma versão final. Como resultado é a proposta da metodologia.

H. Barkhtiary-Dvijany, T.Myhrvold (2013) [10] fizeram uma comparação dos métodos disponíveis de avaliação da maturidade tecnológicas. Os autores criticam a necessidade de analisar a maturidade tecnológica trazendo poucos benefícios em melhorar a tecnologia e propõem um método combinado com uma abordagem baseado em riscos.

A. Aal (2016) [11] Expõe que tecnologias avançadas de semicondutores não são capazes de satisfazer as necessidades da indústria automotiva, propõe um método e testa experimentalmente avaliando o efeito de cargas mecânicas como maneira de simular o envelhecimento desses eletrônicos como um método de projeto voltado a resiliência.

B. Goo et al, (2019) [12] utilizam da ferramenta FMECA (failure mode and criticallity analysis) em conjunto com projeto axiomático para o melhoramento de um sistema de suprimento de gás natural liquefeito. O processo proposto pelo autor é separado em duas fases: a construção do projeto axiomático, como uma etapa conceitual em conjunto com o cliente e aplicar o FMECA para identificação dos riscos e se são riscos que não podem ser ignorados.

S. Yasseri et al, (2018) [13] comparam os estados da prática como as normas API 17 Q e N e as normas DNV-RP-A203 quanto a qualificação tecnológica e propõem um método integrado em utilizar os métodos propostos pelas normas em um processo de desenvolvimento de produto integrado.

Storstenvik (2016) [14] propõe um método modular para qualificação tecnológica e utiliza esse método para um Sistema de compressão subsea, separando o produto a ser qualificado em módulos e utilizando a seguinte sistemática: teste da 
qualificação tecnológica, teste de aceitação pela fábrica, teste funcional e teste de integração.

M. Downes , L. Nguyen (2013) [15] utilizam de ferramentas de confiabilidade para o avanço da maturidade do projeto e separam quais atividades devem ser feitas nas respectivas maturidades tecnológicas. Em uma etapa inicial o processo é análise das funções críticas e testes analíticos e experimentais para a diminuição de incertezas. Em uma etapa em que o Sistema é mais maduro utiliza-se de DOE (Design for experiment) e física da falha para a otimização do projeto. Este estudo utilizou como experimento um fuso aplicado à indústria armamentícia. Algumas ferramentas comuns ao desenvolvimento de produto são utilizadas como o QFD (quality function deployment), que é usado para traduzir as necessidades dos combatentes em requisitos de projetos.

P. Vella et al,(2018) [16] propõem um método para avaliar a maturidade tecnológica de processos micros e nanos de manufatura e parte da seguinte metodologia: definir a escala de maturidade, identificação de indicadores de maturidade, desenvolvimento de questionários para identificação de projetos de pesquisa e desenvolvimento, coleta dos questionários em pesquisadores trabalhando em diferentes projetos no portfólio das empresas, análise dos resultados para obter os esforços necessários da área de pesquisa e desenvolvimento ao longo da escala de maturidade.

T. Albert (2015) [17] propõe um método para avaliar a maturidade tecnológica através do método de blocos de textos de modo rápido, como coleta de dados. $\mathrm{A}$ primeira etapa foi a coleta de informações de tecnologias similares para delinear em relações a tecnologias semelhantes em que será analisada a maturidade para formulação de um questionário preliminar, só então os resultados desses questionários foram confrontados com relação as tecnologias fora da área de pesquisa, para excluir certos termos na pesquisa.

\section{CONCLUSÃO}

Este artigo teve como objetivo identificar a produção científica que aborda a relação de desenvolvimento de produtos e qualificação tecnológica. A pesquisa mostra que a maioria dos estudos utiliza de ferramentas comuns de identificação de riscos, como o FMEA (failure mode analysis) e FTA (fault tree analysis) e poucos desses trabalhos possuem estudos de casos para embasar os resultados e são somente propostas de metodologias.

Por fim, nesta revisão sistemática foi identificado apenas um artigo que leva em consideração necessidades de clientes, sendo somente de clientes internos, mostrando lacunas dos processos de qualificação tecnológica, utilizando de ferramentas de gerenciamento de requisitos para acompanhar o avanço da maturidade tecnológica com o comprimento dos requisitos impostos pelos clientes. 


\section{REFERÊNCIAS}

${ }^{1}$ G. Pahl, W. Beitz, J. Feldhusen, and K.-H. G. Grote, Engineering Design, Third. Darmstadt: Springer Science and Business Media, 2015.

${ }^{2}$ J. Harkonen and J. Hyysalo, "Design for eXcellence in high tech companies Design for eXcellence in high tech companies Keywords : Design for eXcellence , Product development , Requirements," no. December 2008, 2008.

${ }^{3}$ G. L. DNV, “Recommended Practice DNV-RP-203 'Technology Qualification,"” 2013.

${ }^{4}$ P. H. Kim, R. Kotha, S. P. L. Fourné, and K. Coussement, "Taking leaps of faith: Evaluation criteria and resource commitments for early-stage inventions," Res. Policy, vol. 48, no. 6, pp. 1429-1444, 2019.

${ }^{5}$ R. Coelho, R. Daltry, V. Dobbin, E. Lachaud, and I. Miller, "Design Process and Validation of an Autonomous Surface Vehicle for the Offshore Industry," 2015.

${ }^{6}$ E. T. Hessen, H. Bakhtiary-Davijany, and T. Myhrvold, "Process modelling in risk-based qualification of large-scale CO2absorption processes," Energy Procedia, vol. 37, pp. 2802-2810, 2013.

${ }^{7}$ R. Proskovics, G. Hutton, R. Torr, and M. N. Scheu, “Methodology for Risk Assessment of Substructures for Floating Wind Turbines," Energy Procedia, vol. 94, no. January, pp. 45-52, 2016.

${ }^{8} \mathrm{M}$. Sabetzadeh, D. Falessi, L. Briand, and S. Di Alesio, “A goal-based approach for qualification of new technologies: Foundations, tool support, and industrial validation," Reliab. Eng. Syst. Saf., vol. 119, pp. 52-66, 2013.

${ }^{9} \mathrm{~T}$. Myhrvold, K. Helle, K. Johnsen, and A. Hussain, "Development of a guideline for the qualification of CO2 capture technology," Energy Procedia, vol. 1, no. 1, pp. 1527-1534, 2009.

${ }^{10} \mathrm{H}$. Bakhtiary-Davijany and T. Myhrvold, "On methods for maturity assessment of CO2 capture technologies," Energy Procedia, vol. 37, pp. 2579_ 2584, 2013. 
${ }^{11}$ A. Aal, "Reliability management - The central enabler for advanced Technologies in Automotive," Microelectron. Reliab., vol. 64, pp. 13-18, 2016.

${ }^{12}$ B. Goo, J. Lee, S. Seo, D. Chang, and H. Chung, "Design of reliability critical system using axiomatic design with FMECA," Int. J. Nav. Archit. Ocean Eng., vol. 11, no. 1, pp. 11-21, 2019.

${ }^{13}$ S. Yasseri, H. Bahai, and R. Yasseri, "Reliability Assurance of Subsea Production Systems: A Systems Engineering Framework," Int. J. Coast. Offshore Eng., no. January, 2018.

${ }^{14} \mathrm{~A}$. Storstenvik, "Subsea Compression - Designing and Building a Subsea Compressor Station," 2016.

${ }^{15}$ M. R. Downes and L. P. Nguyen, "Reliability engineering efforts at U.S. Army Armaments Research Development and Engineering Center," Proc. - Annu. Reliab. Maintainab. Symp., 2013.

${ }^{16}$ P. C. Vella, S. S. Dimov, R. Minev, and E. B. Brousseau, "Technology maturity assessment of micro and nano manufacturing processes and process chains," Proc. Inst. Mech. Eng. Part B J. Eng. Manuf., vol. 232, no. 8, pp. 1362-1383, 2018.

${ }^{17}$ T. Albert, M. G. Moehrle, and S. Meyer, "Technology maturity assessment based on blog analysis," Technol. Forecast. Soc. Change, vol. 92, pp. 196-209, 2015. 